\title{
Systematic analysis of IncRNA-miRNA- mRNA competing endogenous RNA network identifies four-IncRNA signature as a prognostic biomarker for breast cancer
}

\author{
Chun-Ni Fan, Lei Ma and Ning Liu*
}

\begin{abstract}
Background: Increasing evidence has underscored the role of long non-coding RNAs (IncRNAs) acting as competing endogenous RNAs (ceRNAs) in the development and progression of tumors. Nevertheless, IncRNA biomarkers in IncRNA-related ceRNA network that can predict the prognosis of breast cancer (BC) are still lacking. The aim of our study was to identify potential IncRNA signatures capable of predicting overall survival (OS) of BC patients.

Methods: The RNA sequencing data and clinical characteristics of BC patients were obtained from the Cancer Genome Atlas database, and differentially expressed IncRNA (DEIncRNAs), DEmRNAs, and DEmiRNAs were then identified between BC and normal breast tissue samples. Subsequently, the IncRNA-miRNA-mRNA ceRNA network of $B C$ was established, and the gene oncology enrichment analyses for the DEmRNAs interacting with IncRNAs in the ceRNA network was implemented. Using univariate and multivariate Cox regression analyses, a four-IncRNA signature was developed and used for predicting the survival in BC patients. We applied receiver operating characteristic analysis to assess the performance of our model.

Results: A total of 1061 DElncRNAs, 2150 DEmRNAs, and 82 DEmiRNAs were identified between BC and normal breast tissue samples. A IncRNA-miRNA-mRNA ceRNA network of BC was established, which comprised of 8 DEmiRNAs, 48 DElncRNAs, and 10 DEmRNAs. Further gene oncology enrichment analyses revealed that the DEmRNAs interacting with IncRNAs in the ceRNA network participated in cell leading edge, protease binding, alpha-catenin binding, gamma-catenin binding, and adenylate cyclase binding. A univariate regression analysis of the DElncRNAs revealed 7 IncRNAs (ADAMTS9-AS1, AC061992.1, LINC00536, HOTAIR, AL391421.1, TLR8-AS1 and LINC00491) that were associated with OS of BC patients. A multivariate Cox regression analysis demonstrated that 4 of those IncRNAs (ADAMTS9-AS1, LINC00536, AL391421.1 and LINC00491) had significant prognostic value, and their cumulative risk score indicated that this 4-IncRNA signature independently predicted OS in BC patients. Furthermore, the area under the curve of the 4-IncRNA signature associated with 3-year survival was 0.696.
\end{abstract}

Conclusions: The current study provides novel insights into the IncRNA-related ceRNA network in BC and the 4 IncRNA biomarkers may be independent prognostic signatures in predicting the survival of BC patients.

Keywords: Breast cancer, Long non-coding RNA, Prognosis, Competing endogenous RNA network, Overall survival

*Correspondence: liuning120324@163.com

Department of Breast Surgery, China-Japan Union Hospital of Jilin

University, NO. 126, Xian Tai Street, Changchun 130033, Jilin, China 


\section{Background}

Breast cancer $(\mathrm{BC})$ is a heterogeneous and malignant neoplasm derived from breast tissue, and accounts for about $16 \%$ of all cancers and $22.9 \%$ of invasive cancers in women [1]. The most common cause of the BC-related mortality is metastasis [2, 3]. Currently, $\mathrm{BC}$ diagnosis and prognosis is evaluated on the basis of disease stage, histological grade, and the expression level of hormone receptors [4]. However, clinical and pathological symptoms have limited predictive value in detecting early BC, and the clinical outcomes are highly variable on account of its heterogeneity. In addition, the underlying molecular mechanisms of BC still remain unclear. Therefore, it is vital to identify potential molecular diagnostic markers and/or therapeutic targets to combat $\mathrm{BC}$, especially the invasive form.

Long non-coding RNAs (lncRNAs) is a class of ncRNA over 200 nucleotides long [5], and are reportedly involved in a number of cellular processes, for example, transcriptional and post-transcriptional regulation [5, 6]. Due to their strong tissue specificity, lncRNAs are potentially effective early diagnostic biomarkers of various cancers [7]. Identification of a $\mathrm{BC}$ specific lncRNA biomarker may therefore be of clinical significance for the diagnosis and prognosis of BC. Several lncRNAs have been reported to be associated with $\mathrm{BC}$ initiation and progression $[8,9]$, and although some have been found to predict clinical outcomes for $\mathrm{BC}$, the results are inconsistent due to limited tissue samples. Furthermore, studies without large sample size are also not able to determine with statistical power whether these lncRNAs are associated with survival or other clinical factors. The Cancer Genome Atlas (TCGA) is an open-access and large-scale database which can provide multidimensional molecular profiles for a large number tumor samples. To increase the statistical reliability of our studies, we identified $B C$ specific lncRNAs using data obtained from TCGA database.

The competing endogenous RNA (ceRNA) hypothesis presented by Salmena et al. [10] was proposed as a novel regulatory mechanism between ncRNA and coding messenger RNA. LncRNAs contain miRNA-response elements (MREs) which function as ceRNAs, and play a key role in various pathological processes like tumorigenesis [11]. Zhang et al. [12] have proved the biological role of lncRNA related-ceRNAs in glioblastomas. A recent study has demonstrated that IncRNA NUTF2P3-001 acts as a ceRNA to communicate with KRAS by competitively binding to hsa-mir-3923, and the up-regulation of NUTF2P3-001 reverses the suppressive effect of hsa-mir-3923 on KRAS, leading to the proliferation and invasion of pancreatic cancer [13]. In addition, the aberrant expression of 7-lncRNA signature (called LncRisk-7) led to differential gene expression via a dysregulated
lncRNA-associated ceRNA network, contributing to pancreatic ductal adenocarcinoma progression [14]. Collectively, these findings show that dysregulation of important lncRNAs in the ceRNA network also disrupt the miRNA-mediated lncRNA/mRNA ceRNA interactions and therefore contribute to cancer initiation and progression $[15,16]$. Nevertheless, very little information is available on BC ceRNAs.

In our work, RNA sequencing data of 1109 BC samples and 113 adjacent non-tumor breast tissues samples were retrieved from the TCGA database. To the best of our knowledge, this is the first study to use large scale sequencing database (TCGA) and ceRNA network to identify BC-specific lncRNAs. This new approach of predicting cancer specific lncRNA and ceRNA networks can elucidate the lncRNA-mediated ceRNA regulatory mechanisms in the development and prognosis of $\mathrm{BC}$, and identify novel lncRNAs as potential diagnostic biomarkers or therapeutic targets.

\section{Methods}

Patients and samples from the TCGA database

RNA sequencing (RNA-Seq) data associated with BC were retrieved from the TCGA database (https://porta l.gdc.cancer.gov/, version 10.1, release time: February 15, 2018). A total of 1222 individuals with BC were included in the current study. The exclusion criteria were (1) histological diagnosis negating $\mathrm{BC}$, (2) presence of a malignancy other than $\mathrm{BC}$, and (3) lack of complete clinical data. The gene expression profiles of $1109 \mathrm{BC}$ and 113 adjacent normal breast tissues, and miRNA data of 1103 $\mathrm{BC}$ and 104 adjacent normal breast tissues were downloaded. In addition to the RNA expression data, clinical information of $\mathrm{BC}$ patients were also downloaded from the TCGA database. No approval from the ethics committee was needed because all the information was required from the TCGA database. The clinical characteristics for $\mathrm{BC}$ patients are listed in Table 1.

\section{RNA sequence data processing and differential expression analysis}

The raw RNA sequencing (lncRNA, miRNA, and mRNA) reads were post-processed and normalized using the trimmed mean of M-values (TMM) method.

EdgeR package in R (version 3.4.1) was used to identify the differentially expressed mRNAs (DEmRNAs), lncRNAs (DElncRNAs) and miRNAs (DEmiRNAs) between the $\mathrm{BC}$ and adjacent-normal breast tissues [17], and the cut-off criteria were set as $\mathrm{P}<0.01$ and $|\operatorname{logFC}|>2$. Volcano plots were visualized using the ggplot2packages in $\mathrm{R}$ [18]. The heat map was plotted using the pheatmap function of pheatmap package version 1.0.8 [19]. 
Table 1 The predictive values of clinical features and risk score

\begin{tabular}{|c|c|c|c|c|c|}
\hline \multirow[t]{2}{*}{ Variables } & \multirow[t]{2}{*}{ Patients (N) } & \multicolumn{2}{|c|}{ Univariate analysis } & \multicolumn{2}{|c|}{ Multivariate analysis } \\
\hline & & $\mathrm{HR}(95 \% \mathrm{Cl})$ & $P$ & $\mathrm{HR}(95 \% \mathrm{Cl})$ & $P$ \\
\hline \multicolumn{6}{|l|}{ Age } \\
\hline$<60 / \geq 60$ & $572 / 506$ & $1.67(1.13-2.48)$ & 0.010 & $2.46(1.37-4.42)$ & 0.003 \\
\hline \multicolumn{6}{|l|}{ Pathologic stage } \\
\hline$|-||/|||-\mid V$ & 799/279 & $2.47(1.67--3.66)$ & 0.000 & $2.86(1.15-7.11)$ & 0.024 \\
\hline \multicolumn{6}{|l|}{ Stage $T$} \\
\hline $\mathrm{T} 1-\mathrm{T} 2 / \mathrm{T} 3-\mathrm{T} 4$ & $901 / 177$ & $1.26(0.8-1.97)$ & 0.323 & $1.41(0.59-3.35)$ & 0.440 \\
\hline \multicolumn{6}{|l|}{ Stage N } \\
\hline No/NX & $512 / 566$ & $2.56(1.65-3.95)$ & 0.000 & $1.12(0.53-2.37)$ & 0.773 \\
\hline \multicolumn{6}{|l|}{ Stage M } \\
\hline MO/MX & $901 / 177$ & $1.79(1.05-3.07)$ & 0.034 & $0.48(0.17-1.33)$ & 0.156 \\
\hline \multicolumn{6}{|l|}{ ER } \\
\hline Negative/positive & 232/796 & $0.58(0.38-0.88)$ & 0.011 & $1.15(0.31-4.32)$ & 0.837 \\
\hline \multicolumn{6}{|l|}{ PR } \\
\hline Negative/positive & $338 / 687$ & $0.57(0.38-0.86)$ & 0.007 & $0.54(0.2-1.47)$ & 0.226 \\
\hline \multicolumn{6}{|l|}{ Her2 } \\
\hline Negative/positive & $552 / 163$ & $2.3(1.29-4.1)$ & 0.005 & $2.24(1.06-4.72)$ & 0.034 \\
\hline \multicolumn{6}{|l|}{ Triple negative } \\
\hline No/yes & $598 / 112$ & $1.59(0.82-3.07)$ & 0.168 & $1.69(0.46-6.19)$ & 0.428 \\
\hline \multicolumn{6}{|l|}{ Risk score } \\
\hline Low/high & $593 / 593$ & $2.16(1.42-3.29)$ & 0.000 & $2.26(1.15-4.43)$ & 0.017 \\
\hline
\end{tabular}

$H R$ hazard ratio, $\mathrm{Cl}$ confidence interval

\section{Establishment of the ceRNA network}

The lncRNA-miRNA-mRNA ceRNA network was constructed based on the hypothesis that lncRNAs directly interact with and regulate the activity of mRNAs by acting as miRNA sponges [20]. Based on this hypothesis, we established the IncRNA-miRNA-mRNA ceRNA network in three steps: (1) BC-specific RNAs (lncRNA, mRNA, and miRNA) with $\mathrm{P}<0.01$, and $|\operatorname{logFC}|>2$ were reserved, (2) the potential miRNAs targeted by DElncRNAs and the IncRNA-miRNA interactions were predicted by the miRcode online tool (http://www.mirco de.org), and (3) the MiRDB (http://www.mirdb.org/), miRTarBase (http://mirtarbase.mbc.nctu.edu.tw//), and Targetscan (http://www.targetscan.org//) programs were used to predict the target mRNAs of miRNAs. Finally, the miRNAs that were negatively regulated by the lncRNAs and mRNAs were selected to build the ceRNA network. Cytoscape (version 3.5.1) was used to visualize the lncRNA-miRNA-mRNA ceRNA network.

\section{Functional enrichment analysis}

Gene oncology (GO) is widely used as functional enrichment analysis for a large number of genes [21]. The putative biological roles of DElncRNAs corresponds to that of their associated mRNAs. GO function analyses were therefore conducted for the DEmRNAs in the ceRNA network using $\mathrm{R}$ clusterProfiler package [22]. Fisher's test was used to identify the significant GO terms, and GO categories with $\mathrm{P}<0.05$ were considered statistically significant.

\section{Construction of the BC-specific prognostic signatures}

Kaplan-Meier and log-rank test was used to determine the association between the DEmRNAs, DElncRNAs and DEmiRNAs in the ceRNA network and the overall survival (OS) of BC patients, and statistical significance was set at $\mathrm{P}<0.05$. Univariate Cox proportional hazards regression method was implemented to analyze the relationship between the DElncRNAs and OS when a significant level was set at 0.05 , in order to determine those with a prognostic value in $\mathrm{BC}$. This was followed by multivariate Cox hazards regression model to determine the independent prognostic factors for $\mathrm{BC}$, and the prognostic risk score for predicting OS was as follows:

$$
\begin{aligned}
\text { Risk score }= & \exp _{\text {IncRNA1 }} * \beta_{\text {lncRNA1 }}+\exp _{\text {IncRNA2 } 2} * \beta_{\text {IncRNA2 }} \\
& +\cdots \exp _{\text {IncRNAn }} * \beta_{\text {IncRNAn }}
\end{aligned}
$$

(where "exp" denotes the expression level of DElncRNAs, and " $\beta$ " is the regression coefficient obtained from the multivariate Cox regression model) [23]. Using the median risk score as the threshold, the $\mathrm{BC}$ patients 
were stratified into the high- and low-risk groups. The "survival ROC" package in R was used to construct the time-dependent receiver operating characteristic (ROC) curves within 3 years as the defining point, and to measure the risk prediction rate of specific lncRNAs between the two groups. In addition, the univariate and multivariate analyses were used to evaluate the effects of other clinical variables of $\mathrm{BC}$ patients on OS risk scores. The R software (version 3.4.1) was used for all statistical analyses.

\section{Results}

\section{Identification of DEmRNAs, DElncRNAs, and DEmiRNAs}

We identified the DEmRNAs, DElncRNAs, and DEmiRNAs in BC and adjacent-normal breast tissues using the TCGA database, with $\mathrm{P}<0.01$ and $|\log \mathrm{FC}|>2$ as the thresholds. A total of 2150 DEmRNAs (1368 up- and 782 down-regulated), 1061 DElncRNAs (839 up-, and 222 down-regulated), and 82 DEmiRNAs (62 up- and 20 down-regulated) were identified between $\mathrm{BC}$ and normal samples. Volcano plots displaying the distribution of the DElncRNAs, DEmiRNAs, and DEmRNAs were generated, as shown in Fig. 1a. The heat map showed clear separation and consistency in the expression profiles of the $\mathrm{BC}$ and normal samples (Fig. 1b).

\section{MiRNA predicted target analysis and ceRNA network establishment}

The differentially expressed RNAs identified above were selected, and the lncRNAs and mRNAs targeted by miRNAs were extracted to establish the lncRNA-miRNAmRNA ceRNA network. The relationships among 1061 DElncRNAs and 82 DEmiRNAs were first evaluated. Since lncRNAs might interact with the miRNAs through MREs, the miRcode tool was then used to detect the potential MREs; $18 \mathrm{BC}$-specific miRNAs that putatively target 70 BC-specific lncRNAs were then identified (Additional file 1: Table S1). The MiRDB, miRTarBase and Targetscan programs were then used to determine the relationship between the 82 DEmiRNAs and 2150 DEmRNAs, and predict the mRNA targets of miRNAs. The results indicated that $8 \mathrm{BC}$ specific miRNAs targeted 10 BC-specific mRNAs (Additional file 1: Table S2).

On the basis of the above data, the lncRNA-miRNAmRNA ceRNA network was established and plotted using Cytoscape 3.5.1. Overall, 8 miRNAs (6 up-, and 2 down-regulated, Table 2), 48 lncRNAs (32 up-, and 16 down-regulated, Table 3), and 10 mRNAs ( 2 up-, and 8 down-regulated, Table 4) were involved in the proposed ceRNA network (Fig. 2). Based on the expression levels of DEmRNAs, DElncRNAs and DEmiRNAs, two ceRNA networks including under-expressed (Fig. 2a) and overexpressed (Fig. 2b) networks were constructed.

\section{Delineation of GO analysis}

In order to better understand the role of the DElncRNAs in $\mathrm{BC}$, we analyzed the mRNAs of the ceRNA network and identified the lncRNA regulated GO terms. The results of GO analysis are shown in Additional file 2: Figure S1. Our data showed that the mRNAs associated to cellular component (CC), was cell leading edge. Ge et al. [24] have demonstrated that trypsin secreted from MDA MB-231 BC cells activates the protease-activated receptor- 2 and the activated protease-activated receptor-2 can promote cell migration based on ERK1/2-dependent pathway, involving the formation of a scaffolding complex at the cell leading edge. Meanwhile, the mRNAs related to molecular function (MF) were most relevant to protease binding, alpha-catenin binding, gamma-catenin binding, and adenylate cyclase binding. Proteases provide the cancer a characteristic of being able to invade into other tissues, and protease-activated receptor- 1 is involved in the migration and invasion of breast cancer cells [25]. The loss of alpha-catenin has been implicated to be related to the metastasis and poor survival in BC [26]. The expression of gamma-catenin was also reported to be associated with the metastasis in human $\mathrm{BC}$ [27]. The activity of the adenylate cyclase is positively linked to the inhibition of cell proliferation, as well as induction of apoptosis in human BC MCF-7 cells [28]. Demonstrated herein, these $\mathrm{GO}$ terms are associated with $\mathrm{BC}$ pathogenesis and prognosis.

\section{Correlations between BC specific signatures and OS}

Kaplan-Meier and log-rank test were used to determine the relationship between the DEmRNAs, DElncRNAs and DEmiRNAs in the ceRNA network and the OS of $B C$ patients with a cut-off threshold of $\mathrm{P}<0.05$. Totally, 4 DElncRNAs (ADAMTS9-AS1, AL356479.1, CHL1-AS2, and LINC00536; Fig. 3a), 3 DEmiRNAs (hsa-miR-204, hsa-miR-210, and hsa-miR-429; Fig. 3b), and 1 DEmRNA (KPNA2; Fig. 3c) were found to be related to OS.

\section{Establishment of the 4-IncRNAs prognostic model}

Univariate regression analysis was used to identify the IncRNAs associated with the OS of BC patients. With the significance level cutoff threshold set at $\mathrm{P}<0.05$, a group of lncRNA signatures including ADAMTS9-AS1, AC061992.1, LINC00536, HOTAIR, AL391421.1, TLR8AS1, and LINC00491 lncRNAs was detected to have significant prognostic value (Additional file 1: Table S3). Significantly, we found that ADAMTS9-AS1 was simultaneously identified to be connected with OS in KaplanMeier (log-rank test) and univariate Cox regression analysis. 

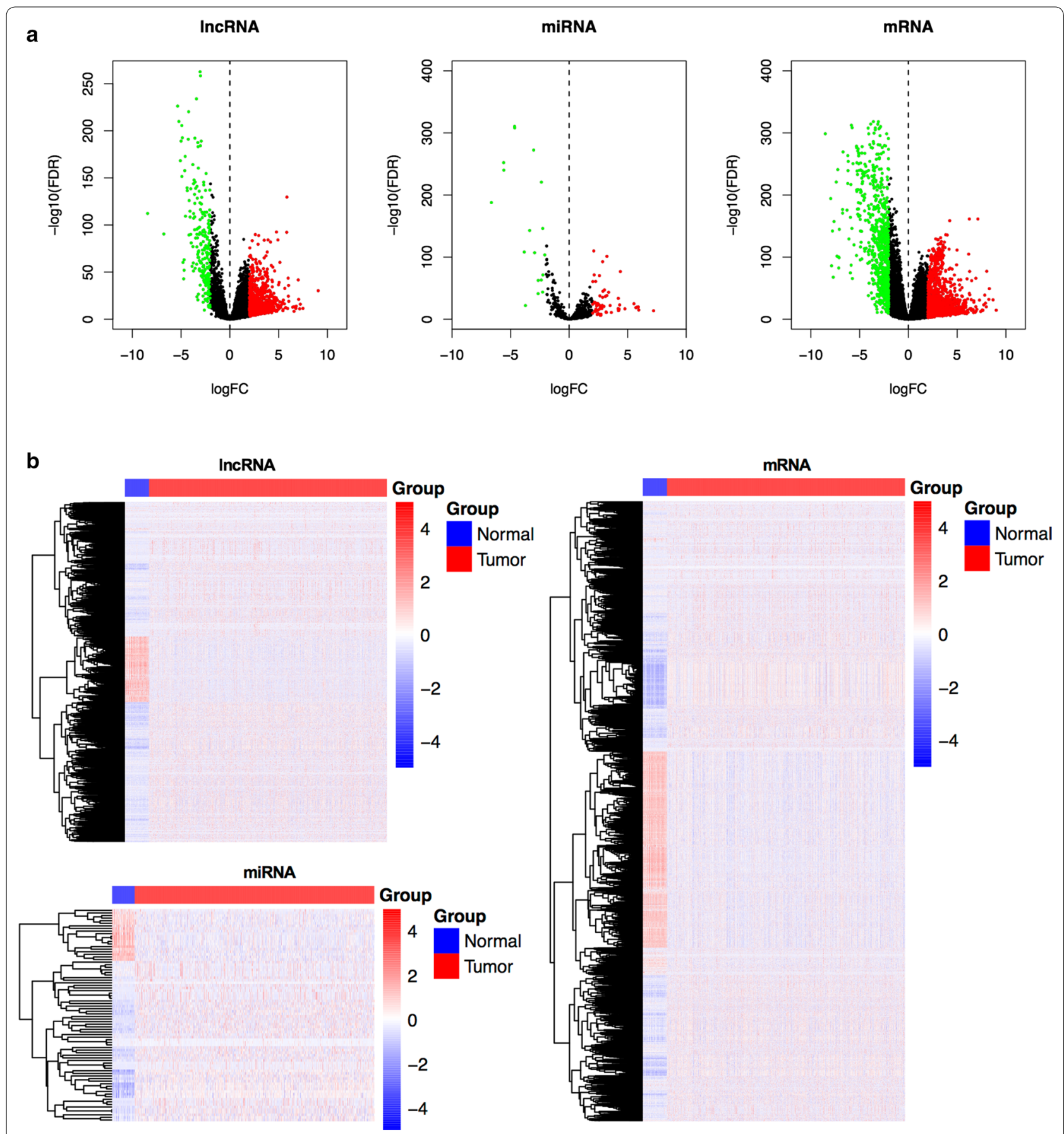

Fig. 1 a Volcano plots showing the differential expression of RNAs (IncRNAs, miRNAs, and mRNAs) in breast cancer (BC), which were drawn using the $R$ packages ggplot2; $X$ axis indicates the mean expression differences of IncRNAs, miRNAs, and mRNAs between BC and normal samples, and $Y$ axis represents log transformed false discovery rate (FDR) values. $\mathbf{b}$ Heatmaps demonstrate differential expression of IncRNAs, miRNAs, and mRNAs between $B C$ and adjacent normal samples, which were plotted using the pheatmap package; $X$ axis denotes differentially expressed IncRNAs (DElncRNAs), DEmiRNAs, and DEmRNAs and Y axis represents the samples. Blue represents normal samples, while red stands for BC samples. The expression values are shown in line with the color scale 
Table 2 miRNAs in ceRNA network of BC

\begin{tabular}{llll}
\hline miRNA & logFC & $\boldsymbol{P}$ value & FDR \\
\hline hsa-mir-210 & 3.022945333 & $5.83 \mathrm{E}-49$ & $7.72 \mathrm{E}-48$ \\
hsa-mir-183 & 2.861143375 & $8.29 \mathrm{E}-95$ & $2.30 \mathrm{E}-93$ \\
hsa-mir-429 & 2.590469156 & $3.89 \mathrm{E}-72$ & $7.09 \mathrm{E}-71$ \\
hsa-mir-137 & 2.481127225 & $4.30 \mathrm{E}-10$ & $9.52 \mathrm{E}-10$ \\
hsa-mir-182 & 2.265833003 & $1.04 \mathrm{E}-62$ & $1.64 \mathrm{E}-61$ \\
hsa-mir-21 & 2.105244174 & $2.87 \mathrm{E}-112$ & $1.19 \mathrm{E}-110$ \\
hsa-mir-204 & -2.643779105 & $2.00 \mathrm{E}-64$ & $3.23 \mathrm{E}-63$ \\
hsa-mir-144 & -2.948045408 & $2.31 \mathrm{E}-109$ & $8.42 \mathrm{E}-108$ \\
\hline
\end{tabular}

All the above lncRNAs were then fitted into the multivariate Cox regression model, which indicated that only four lncRNAs-ADAMTS9-AS1, LINC00536, AL391421.1 and LINC00491-had a significant prognostic value in $\mathrm{BC}$ (Additional file 3: Figure S2C), and these four lncRNAs were used to develop an IncRNA prognostic model. A risk score analysis of the four lncRNAs was performed for each patient, and based on the risk scores, the patients were divided into the "low risk" and "high risk" groups (Additional file 3: Figure S2A). The mortality rate of the high risk patients was significantly higher compared to the low risk patients $(12.99 \%$ vs $5.94 \%$; $\mathrm{P}<0.05$; Additional file 3: Figure S2B).

In addition, the high risk group was correlated with worse prognosis compared to the low risk group (Fig. 4a). The 3 year survival correlation of the 4-lncRNA signature was analyzed by ROC and AUC was computed to assess the discriminatory capacity of lncRNA signature (Fig. 4b). The AUC of the 4-lncRNA signature was 0.696 indicating its utility as a prognostic model for predicting the survival status of BC.

The 4-IncRNA expression was then analyzed in the tumor and normal tissues, and in the high- and lowrisk patient groups (Fig. 5). ADAMTS9-AS1, and AL391421.1 were expressed at high levels in patients with low-risk scores, whereas LINC00536 and LINC00491 were up-regulated in the high-risk patients. Furthermore, LINC00491, AL391421.1, and LINC00536 were expressed at high levels, and ADAMTS9-AS1 was expressed at low levels in the $\mathrm{BC}$ patients.

\section{Prognostic value of the four-IncRNA signature in BC}

Univariate and multivariate regression models were used to assess the prognostic power of the 4-IncRNA signature. Univariate analysis indicated that age, pathological stage, $\mathrm{N}$ stage, $\mathrm{M}$ stage, ER, PR, Her2, and risk scores were significantly correlated with OS of BRCA patients $(\mathrm{P}<0.05)$. Similarly, Kaplan-Meier analysis demonstrated
Table 3 IncRNA in ceRNA network of BC

\begin{tabular}{|c|c|c|c|}
\hline IncRNA & $\log \mathrm{FC}$ & $P$ value & FDR \\
\hline DSCAM-AS1 & 5.99941085 & $4.44 \mathrm{E}-26$ & $4.13 \mathrm{E}-25$ \\
\hline LINC00261 & 5.43693494 & $3.30 \mathrm{E}-10$ & $9.98 \mathrm{E}-10$ \\
\hline LINC00305 & 5.05852227 & $2.85 E-13$ & $1.14 \mathrm{E}-12$ \\
\hline ATXN8OS & 4.46010816 & $3.17 \mathrm{E}-11$ & $1.06 \mathrm{E}-10$ \\
\hline LINC00221 & 4.39970195 & $1.02 \mathrm{E}-12$ & $3.88 \mathrm{E}-12$ \\
\hline LINC00210 & 4.30883058 & $4.11 \mathrm{E}-11$ & $1.36 \mathrm{E}-10$ \\
\hline LINC00466 & 4.24876959 & $2.08 \mathrm{E}-59$ & $7.81 \mathrm{E}-58$ \\
\hline LINC00518 & 4.15626859 & $1.43 \mathrm{E}-15$ & $6.88 \mathrm{E}-15$ \\
\hline MIR7-3HG & 4.10845802 & $9.51 \mathrm{E}-14$ & $3.95 \mathrm{E}-13$ \\
\hline MUC19 & 4.07948976 & $3.60 \mathrm{E}-20$ & $2.36 \mathrm{E}-19$ \\
\hline LINC00461 & 3.60834524 & $3.96 \mathrm{E}-19$ & $2.43 \mathrm{E}-18$ \\
\hline AL589642.1 & 3.54021424 & $2.88 \mathrm{E}-10$ & $8.74 \mathrm{E}-10$ \\
\hline C2orf48 & 3.44559688 & $6.13 \mathrm{E}-56$ & $2.05 \mathrm{E}-54$ \\
\hline LINC00200 & 3.39237322 & $5.31 \mathrm{E}-09$ & $1.43 \mathrm{E}-08$ \\
\hline AL391421.1 & 3.26256052 & $3.52 \mathrm{E}-28$ & $3.66 \mathrm{E}-27$ \\
\hline HOTAIR & 2.99777460 & $1.71 \mathrm{E}-29$ & $1.92 \mathrm{E}-28$ \\
\hline CLRN1-AS1 & 2.96319950 & $3.12 \mathrm{E}-29$ & $3.42 \mathrm{E}-28$ \\
\hline SMCR2 & 2.94279962 & $3.39 \mathrm{E}-45$ & $7.75 \mathrm{E}-44$ \\
\hline TCL6 & 2.90585029 & $2.21 \mathrm{E}-21$ & $1.57 \mathrm{E}-20$ \\
\hline LINC00536 & 2.82907445 & $1.74 \mathrm{E}-33$ & $2.43 \mathrm{E}-32$ \\
\hline LINC00524 & 2.61155065 & $7.48 \mathrm{E}-15$ & $3.41 \mathrm{E}-14$ \\
\hline LINC00488 & 2.59389493 & $3.24 \mathrm{E}-08$ & $8.12 \mathrm{E}-08$ \\
\hline FNDC1-IT1 & 2.36336842 & $3.22 \mathrm{E}-22$ & $2.41 \mathrm{E}-21$ \\
\hline C10orf91 & 2.33148238 & $1.26 \mathrm{E}-24$ & $1.09 \mathrm{E}-23$ \\
\hline MAST4-IT1 & 2.28036554 & 1.05E-07 & $2.51 \mathrm{E}-07$ \\
\hline TLR8-AS1 & 2.26971966 & $2.22 \mathrm{E}-11$ & $7.45 \mathrm{E}-11$ \\
\hline C1orf137 & 2.26365184 & $3.59 \mathrm{E}-12$ & $1.29 \mathrm{E}-11$ \\
\hline AC061992.1 & 2.24485511 & $7.75 E-45$ & $1.75 E-43$ \\
\hline SHANK2-AS3 & 2.15540014 & 1.36E-08 & $3.53 \mathrm{E}-08$ \\
\hline AC127496.1 & 2.09598212 & $6.09 \mathrm{E}-30$ & $7.02 \mathrm{E}-29$ \\
\hline LINC00491 & 2.05604565 & 7.95E-06 & $1.59 \mathrm{E}-05$ \\
\hline DLX6-AS1 & 2.019341904 & $6.94 \mathrm{E}-11$ & $2.25 \mathrm{E}-10$ \\
\hline EMX2OS & -2.12877927 & $6.46 \mathrm{E}-83$ & $4.40 \mathrm{E}-81$ \\
\hline PWRN1 & -2.18494962 & $4.42 \mathrm{E}-16$ & $2.21 \mathrm{E}-15$ \\
\hline CHL1-AS1 & -2.32544868 & $1.99 \mathrm{E}-39$ & $3.63 \mathrm{E}-38$ \\
\hline C20orf166-AS1 & -2.40983541 & $4.19 \mathrm{E}-43$ & $8.75 \mathrm{E}-42$ \\
\hline PHEX-AS1 & -2.41002550 & $4.36 \mathrm{E}-29$ & $4.73 E-28$ \\
\hline AL356479.1 & -2.47223771 & $1.01 \mathrm{E}-37$ & $1.72 \mathrm{E}-36$ \\
\hline RBMS3-AS3 & -2.55564241 & $5.60 E-113$ & $8.32 \mathrm{E}-111$ \\
\hline AGAP11 & -2.57967944 & $1.99 \mathrm{E}-116$ & $3.36 \mathrm{E}-114$ \\
\hline AC040173.1 & -2.62010067 & $2.11 \mathrm{E}-41$ & $4.18 \mathrm{E}-40$ \\
\hline ADAMTS9-AS1 & -2.65113038 & $4.59 \mathrm{E}-96$ & $4.63 \mathrm{E}-94$ \\
\hline ARHGEF7-AS2 & -2.77120520 & $1.03 \mathrm{E}-114$ & $1.61 \mathrm{E}-112$ \\
\hline CHL1-AS2 & -2.80546689 & $7.46 \mathrm{E}-76$ & $4.28 \mathrm{E}-74$ \\
\hline MME-AS1 & -3.01455968 & $2.41 \mathrm{E}-62$ & $9.89 \mathrm{E}-61$ \\
\hline ADAMTS9-AS2 & -3.02086308 & $1.16 \mathrm{E}-187$ & $7.25 \mathrm{E}-185$ \\
\hline ALDH1L1-AS2 & -4.86910828 & $2.30 \mathrm{E}-196$ & $2.53 \mathrm{E}-193$ \\
\hline ADIPOQ-AS1 & -5.07515076 & $1.06 \mathrm{E}-171$ & $5.48 \mathrm{E}-169$ \\
\hline
\end{tabular}


Table 4 mRNA in ceRNA network of BC

\begin{tabular}{llll}
\hline mRNA & logFC & P value & FDR \\
\hline CDH2 & 2.57235331 & $7.09 E-27$ & $3.37 \mathrm{E}-26$ \\
KPNA2 & 2.14681192 & $3.85 \mathrm{E}-77$ & $7.06 \mathrm{E}-76$ \\
SH3D19 & -2.0172533 & $2.91 \mathrm{E}-244$ & $4.34 \mathrm{E}-242$ \\
TCEAL7 & -2.1550474 & $8.72 \mathrm{E}-128$ & $3.65 \mathrm{E}-126$ \\
WASF3 & -2.1800702 & $6.27 \mathrm{E}-121$ & $2.38 \mathrm{E}-119$ \\
SPRY2 & -2.4420515 & $6.19 \mathrm{E}-188$ & $5.38 \mathrm{E}-186$ \\
AKAP12 & -2.7016705 & $6.57 \mathrm{E}-188$ & $5.69 \mathrm{E}-186$ \\
CHL1 & -2.9649624 & $5.86 \mathrm{E}-121$ & $2.23 \mathrm{E}-119$ \\
KIT & -2.9705757 & $7.12 \mathrm{E}-126$ & $2.85 \mathrm{E}-124$ \\
SERTM1 & -3.6706976 & $2.69 \mathrm{E}-84$ & $5.55 \mathrm{E}-83$ \\
\hline
\end{tabular}

that clinical factors (age, pathological stage, $\mathrm{N}$ stage, $\mathrm{M}$ stage, ER, PR, and Her2) were significantly correlated to OS, which was consistent with the univariate analysis. Kaplan-Meier curves of the clinical characteristics are shown in Fig. 6. Multivariate analysis indicated that only age, pathological stage, Her2, and risk scores were independent prognostic factors of $\mathrm{OS}(\mathrm{P}=0.003,0.024,0.034$, and 0.017 respectively; Table 1 ).

\section{Discussion}

$\mathrm{BC}$ is a common malignant gynecological cancer, and is one of the main causes for the cancer-related deaths in women [29]. The lack of specific diagnostic and prognostic biomarkers may contribute to the current low survival rate among $\mathrm{BC}$ patients. To improve clinical outcomes therefore, it is essential to explore the exact regulatory mechanisms of $\mathrm{BC}$ initiation and progression, and to identify the potential $\mathrm{BC}$-related prognostic signatures that predict those outcomes. Growing experimental evidence indicates that lncRNAs play important roles in many biological processes, and ceRNA activity is closely related to the development of cancers [30,31].

In recent years, some studies have investigated the ceRNAs in BC. For instance, Chen et al. [32] analyzed the $B C$ ceRNA network on the basis of common miRNAs as well as co-expression, but did not consider miRNA expression. Another study also established a BC specific ceRNA network to investigate its underlying molecular mechanisms based on the PCC of miRNAmRNA pairs [33]. However, both studies focused on the roles of mRNAs rather than that of lncRNAs in the BC ceRNA networks. In 2018, Zhou et al. [34] constructed four BC-related ceRNA networks by combining the miRNA targets and the expression data of lncRNAs, miRNAs and mRNA, but they did not take into account the relationship between survival and lncRNAs, nor construct the prognostic signature. In our study, in addition to constructing the ceRNA networks by combining lncRNA, miRNA, and mRNA expression data, we also investigated the association of lncRNA and $\mathrm{OS}$ in $\mathrm{BC}$ patients. Furthermore, based on the theory of ceRNA network, we established the 4-lncRNAs

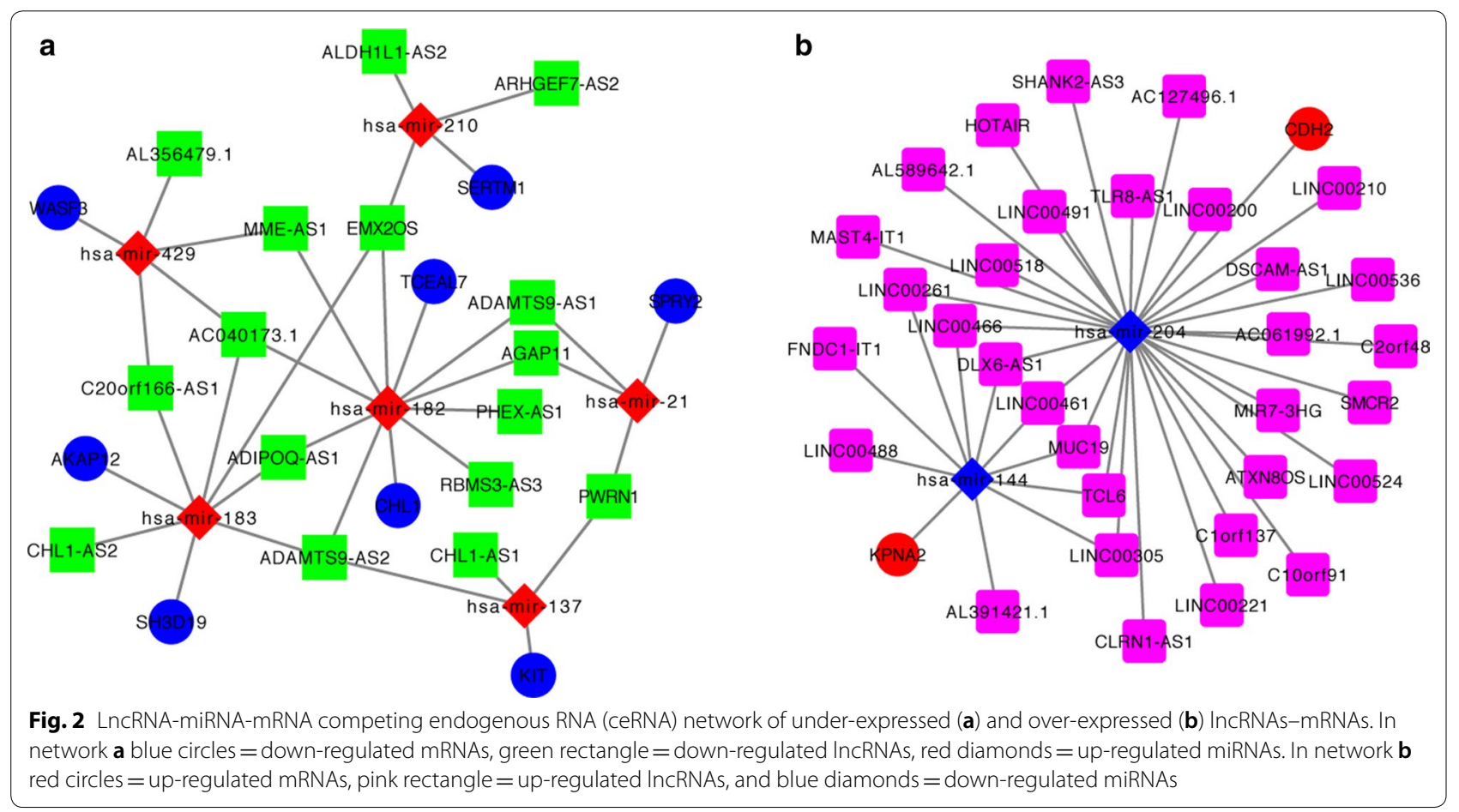



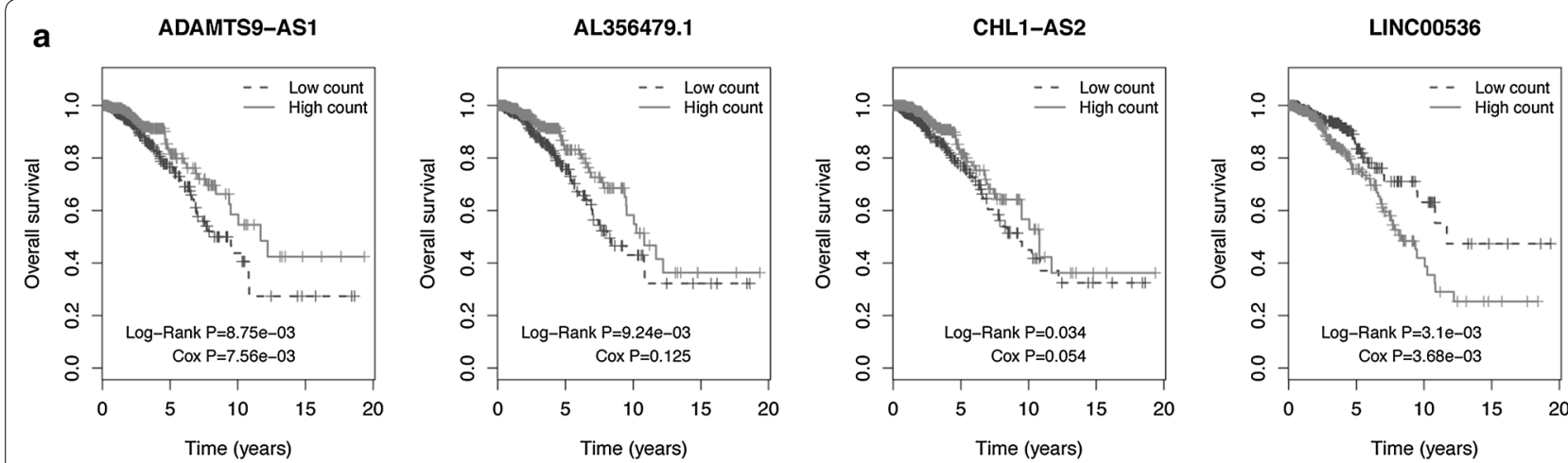

b
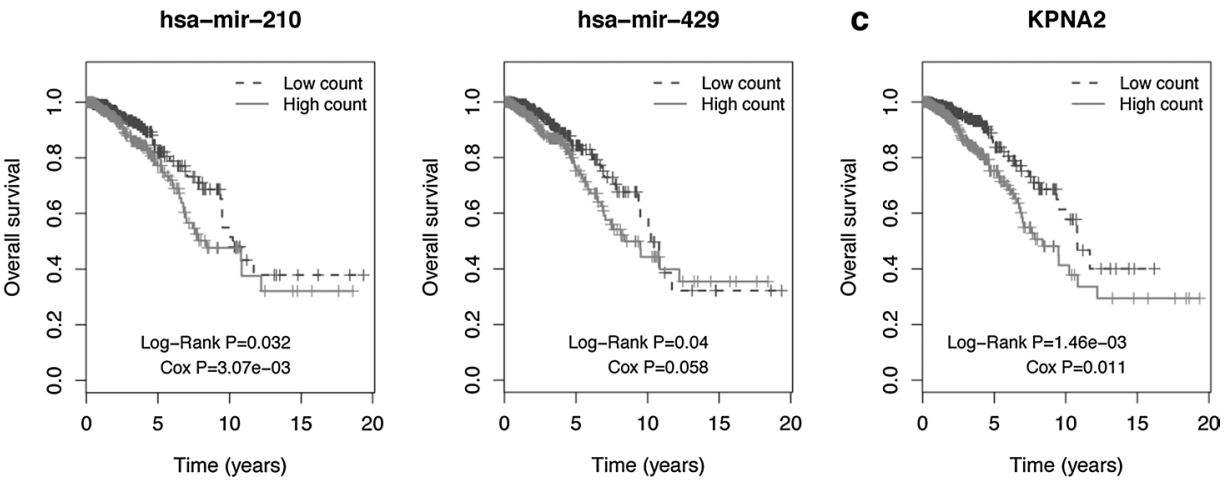

Fig. 3 Kaplan-Meier survival curves for a DEIncRNAs, b DEmiRNAs, and $\mathbf{c}$ DEmRNAs associated with overall survival (OS) of the BC patients. Log-rank method was used to assess the survival differences between the two groups. Horizontal axis is OS time (years) and vertical axis stands for survival function
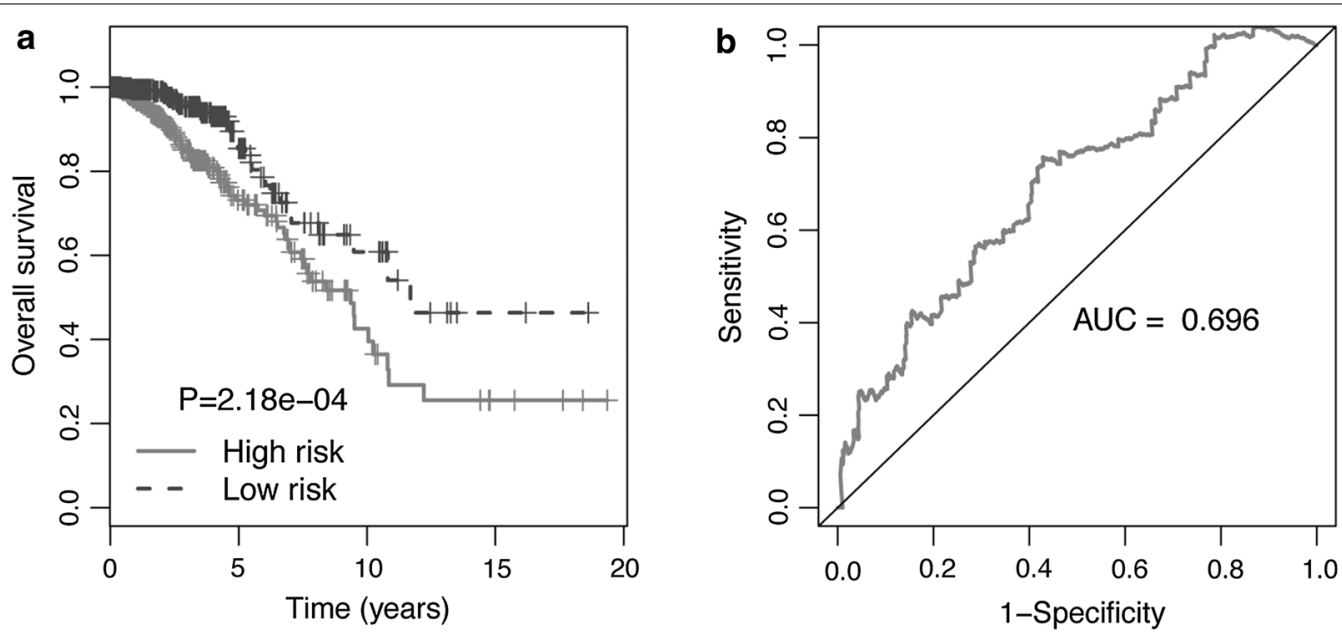

Fig. 4 The 4-InCRNA signature of BC (ADAMTS9-AS1, AL391421.1, LINC00491, and LINC00536) for the outcome. a The survival differences between the high-risk and low-risk groups were determined by the log-rank test $(P=2.18 \mathrm{E}-04)$. $\mathbf{b} \mathrm{ROC}$ curves demonstrated that the area under receiver operating characteristic (AUC) of 4-IncRNA model was 0.696, which exhibited the risk score

prognostic signature. With the goal of identifying lncRNAs significantly associated with OS, we established an IncRNA-miRNA-mRNA ceRNA network using the information obtained from the TCGA database.
Univariate regression analysis on the DElncRNAs of the ceRNA network identified 7 lncRNAs-ADAMTS9AS1, AC061992.1, LINC00536, HOTAIR, AL391421.1, TLR8-AS1 and LINC00491-that were associated with 

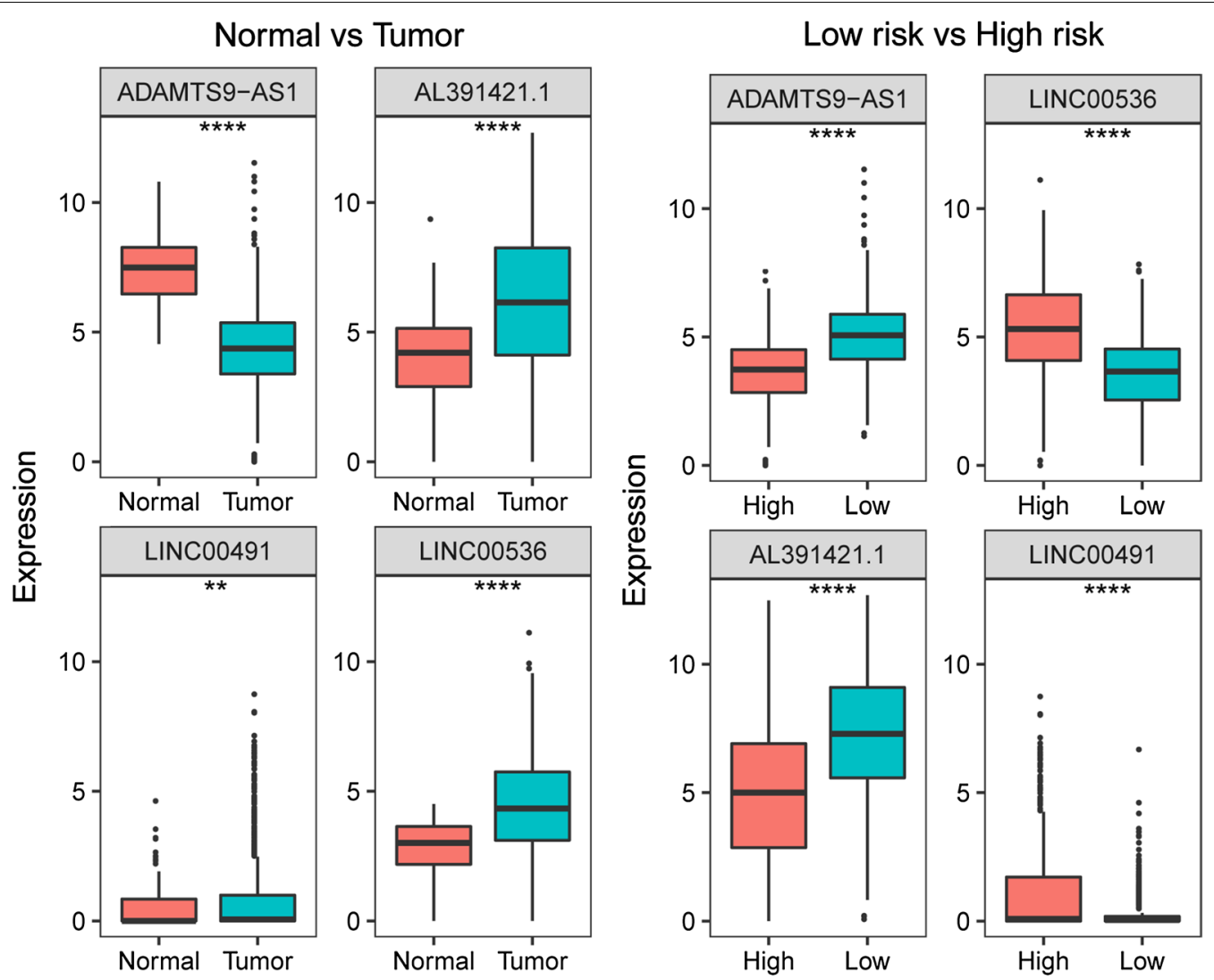

Fig. 5 Expression pattern of the 4-InCRNA (ADAMTS9-AS1, AL391421.1, LINC00491, and LINC00536) in BC and normal tissues, and in high-risk and low-risk groups

OS. Multivariate analysis showed significant prognostic value of 4 of those lncRNAs (ADAMTS9-AS1, LINC00536, AL391421.1 and LINC00491) in the OS of BC patients. A cumulative risk score of the 4 lncRNAs was calculated, which indicated that this 4-lncRNA signature independently predicted OS in BC patients. To the best of our knowledge, this is the first report integrating a ceRNA network with TCGA data to build an lncRNA-related risk score, and evaluate the OS of BC patients. Our study will help improve the understanding of lncRNA-mediated ceRNA regulatory mechanisms in BC and identify novel lncRNAs as therapeutic targets.

In the current study, among this 4-lncRNA signature, ADAMTS9-AS1 was demonstrated to play important roles in the progression and prognosis of cancer. ADAMTS9-AS1 is an antisense lncRNA, and growing evidence has implicated that a large amount of antisense lncRNAs play crucial roles in the cancer [35, 36]. Li et al. [37] reported that ADAMTS9-AS1 could predict the survival status of patients with esophageal squamous cell carcinoma. Another study has also indicated a prognostic role of ADAMTS9-AS1 in patients with colon adenocarcinoma [38]. In addition, ADAMTS9-AS1 has been demonstrated to be a risk lncRNA in ovarian cancer, which is involved in the progression of ovarian cancer [39]. In our study, we noticed that ADAMTS9-AS1 with lowexpression could compete with up-regulated miRNAs (hsa-mir-182, and hsa-mir-21), to regulate the expression of the target genes such as CHL1, SPRY2, and TCEAL7 involved in the ceRNA network. Previous studies have shown high expression of hsa-mir-182 in MCF-7 breast cancer cells [40, 41]. In addition, the high-expression of hsa-mir-21 was reported to be correlated to the metastasis and poor prognosis of $\mathrm{BC}$ patients [42]. The remaining three lncRNAs of the ceRNA network (LINC00536/ AL391421.1/LINC00491) were up-regulated and competed with the decreased hsa-mir-204 and hsa-mir-144 levels. Down-regulation of has-mir-204 has been suggested to enhance cell proliferation and invasion in gastric cancer [43], and low-expression of has-mir-204 is related to the poor prognosis of acute myeloid leukemia patients [44]. In addition, decreased expression of hasmir-144 is strongly correlated with the progression of 


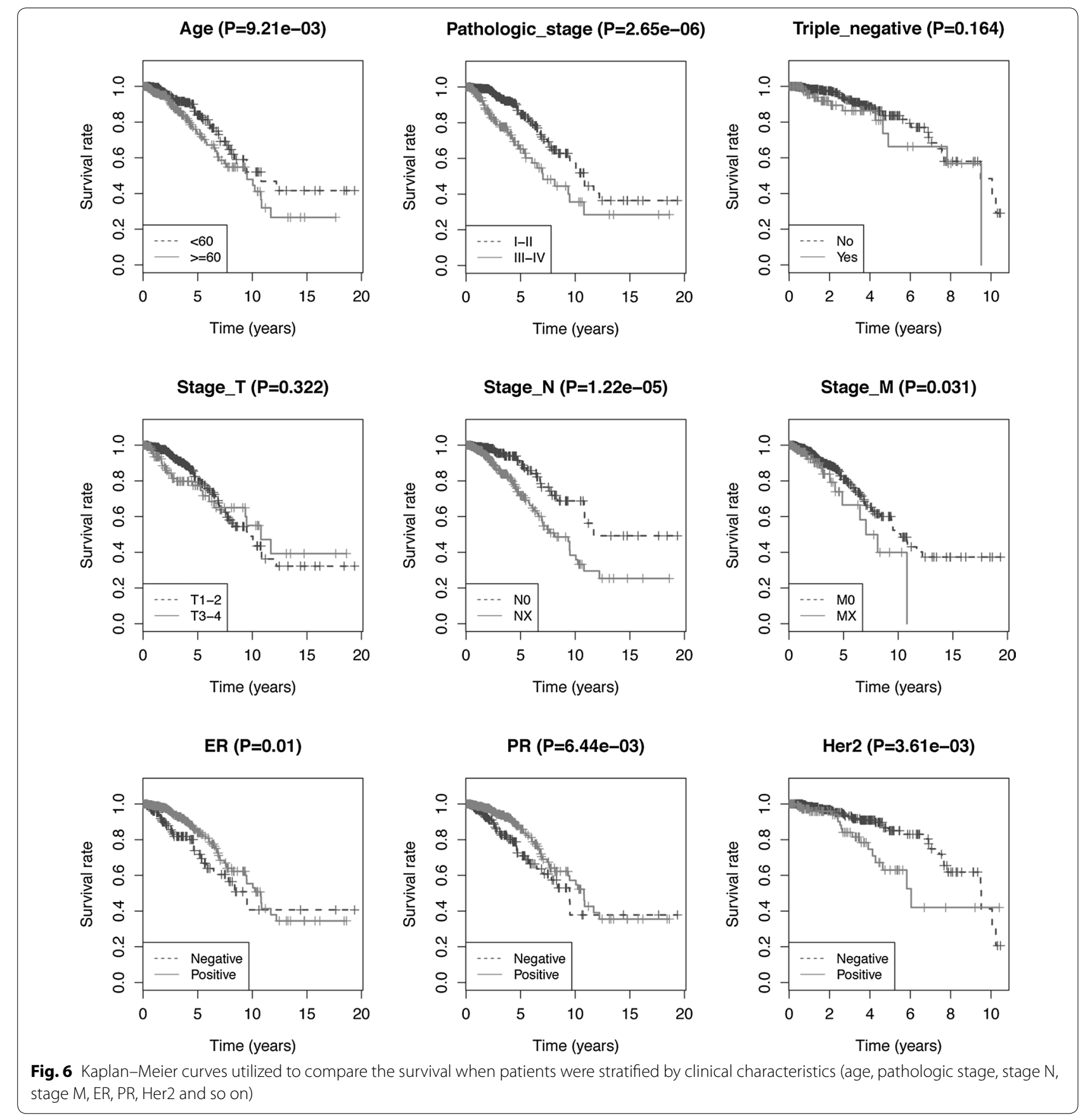

colorectal cancer [45]. No study so far has reported any association of LINC00536, AL391421.1 or LINC00491 with cancer. This is the first study to show aberrant expression of ADAMTS9-AS1, LINC00536, AL391421.1 and LINC00491 in BC, and indicates a potential prognostic role of this 4-lncRNA signature in BC. In addition, the bioinformatics based investigation of lncRNAs will be helpful in future experimental studies.
Although the findings of our study have important clinical implications, the limitations must also be noted. First, a longer follow-up duration is required to verify our results, and second, the findings based on the TCGA database will need to be verified using other experimental methods. In addition, the biological roles of ADAMTS9AS1, LINC00536, AL391421.1, and LINC00491 in BC also need to be further investigated. 


\section{Conclusion}

Taken together, we have identified a 4-lncRNA signature as a potential prognostic predictor for BC patients by analyzing the genome-wide lncRNA expression data from the TCGA database based on a ceRNA network. The current findings provide novel insights into the lncRNA-related ceRNA network in BC and identify potential diagnostic and prognostic biomarkers. Further functional studies are needed to elucidate the molecular mechanisms underlying lncRNA function in BC.

\section{Additional files}

Additional file 1. miRNAs targeting IncRNAs and mRNAs ofBC, as well as the prognostic value of the IncRNAs obtained from the univariate Cox's analysis.

Additional file 2: Figure S1. Gene Ontology (GO) analysis. GO results for aberrantly expressed mRNAs with significant Enrichment score covering domains of cellular components (CCS) and molecular functions (MFs). The bar plot devotes the enrichment scores of the significant $\mathrm{GO}$ terms.

Additional file 3: Figure S2. Four-InCRNA signature ((ADAMTS9-AS1, AL391421.1, LINC00491, and LINC00536)) predicted OS in BC cohort. A. Risk-score distribution. Red demonstrating higher expression while blue representing lower expression. Risk scores for all BC patients were created in ascending order and blue is marked as low risk or red is labeled as high risk. B. Patients' survival status with blue devoting dead, and red standing for alive. C. Heat map of the four-IncRNA expression profiles in BC patients.

\section{Abbreviations}

IncRNAs: long non-coding RNAs; ceRNAs: competing endogenous RNAs; BC: breast cancer; OS: overall survival; TCGA: the Cancer Genome Atlas; DElncRNAs: differentially expressed IncRNA; MREs: miRNA-response elements; GO: gene oncology; ROC: receiver operating characteristic; CC: cellular component:; MF: molecular function.

\section{Authors' contributions}

CNF and LM conceived and designed the experiments. CNF, LM and NL analyzed data. LM and NL wrote this manuscript. All authors read and approved the final manuscript.

\section{Acknowledgements}

None.

\section{Competing interests}

The authors declare that they have no competing interests.

\section{Availability of data and materials}

Not applicable.

\section{Consent for publication}

Not applicable.

Ethics approval and consent to participate

Not applicable.

\section{Funding}

None.

\section{Publisher's Note}

Springer Nature remains neutral with regard to jurisdictional claims in published maps and institutional affiliations.
Received: 28 May 2018 Accepted: 20 September 2018

Published online: 27 September 2018

\section{References}

1. Siegel R, Naishadham D, Jemal A. Cancer statistics, 2013. CA Cancer J Clin. 2013;63(1):11.

2. Hanahan D, Weinberg RA. Hallmarks of cancer: the next generation. Cell. 2011;144(5):646.

3. Gupta GP, Massagué J. Cancer metastasis: building a framework. Cell. 2006;127(4):679.

4. Vuong D, et al. Molecular classification of breast cancer. Virchows Archiv. 2014;465(1):1-14

5. Mercer TR, Dinger ME, Mattick JS. Long non-coding RNAs: insights into functions. Nat Rev Genet. 2009;10(3):155-9.

6. Fatica A, Bozzoni I. Long non-coding RNAs: new players in cell differentiation and development. Nat Rev Genet. 2014;15(1):7-21.

7. Hung T, Chang HY. Long noncoding RNA in genome regulation: prospects and mechanisms. RNA Biol. 2010;7(5):582.

8. Su X, et al. Comprehensive analysis of long non-coding RNAs in human breast cancer clinical subtypes. Oncotarget. 2014:5(20):9864.

9. Jin $\mathrm{M}$, et al. A four-long non-coding RNA signature in predicting breast cancer survival. J Exp Clin Cancer Res. 2014:33(1):84.

10. Salmena $L$, et al. ceRNA hypothesis: the Rosetta Stone of a hidden RNA language? Cell. 2011;146(3):353-8.

11. Song $X$, et al. Analysing the relationship between IncRNA and proteincoding gene and the role of IncRNA as ceRNA in pulmonary fibrosis. J Cell Mol Med. 2014;18(6):991-1003.

12. Zhang $K$, et al. Identification and functional characterization of IncRNAs acting as ceRNA involved in the malignant progression of glioblastoma multiforme. Oncol Rep. 2016;36(5):2911-25.

13. Xiang $L$, et al. Hypoxia-induced IncRNA-NUTF2P3-001 contributes to tumorigenesis of pancreatic cancer by derepressing the miR-3923/KRAS pathway. Oncotarget. 2016;7(5):6000-14.

14. Zhou M, et al. Construction and analysis of dysregulated IncRNAassociated ceRNA network identified novel IncRNA biomarkers for early diagnosis of human pancreatic cancer. Oncotarget. 2016;7(35):56383-94.

15. Karreth FA, Pandolfi PP. ceRNA cross-talk in cancer: when ce-bling rivalries go awry. Cancer Discov. 2013;3(10):1113-21.

16. Tan JY, et al. Extensive microRNA-mediated crosstalk between IncRNAs and mRNAs in mouse embryonic stem cells. Genome Res. 2015;25(5):655.

17. Robinson MD, McCarthy DJ, Smyth GK. EdgeR: a bioconductor package for differential expression analysis of digital gene expression data. Bioinformatics. 2009;26(1):139-40.

18. Wickham H. ggplot2: elegant graphics for data analysis. Houston: Springer Publishing Company, Incorporated; 2009. p. 180-5.

19. Kolde R. pheatmap: pretty heatmaps; 2015.

20. Guo LL, et al. Competing endogenous RNA networks and gastric cancer. World J Gastroenterol. 2015;21(41):11680.

21. Ashburner M, et al. Gene ontology: tool for the unification of biology. Gene ontology consortium. Nat Genet. 2000;25(1):25-9.

22. Yu G, et al. clusterProfiler: an $\mathrm{R}$ package for comparing biological themes among gene clusters. Omics-a J Integr Biol. 2012;16(5):284-7.

23. Zeng $J \mathrm{H}$, et al. Comprehensive investigation of a novel differentially expressed IncRNA expression profile signature to assess the survival of patients with colorectal adenocarcinoma. Oncotarget. 2017;8(10):16811.

24. Ge L, et al. Constitutive protease-activated receptor-2-mediated migration of MDA MB-231 breast cancer cells requires both beta-arrestin-1 and -2. J Biol Chem. 2004;279(53):55419-24.

25. Kamath $L$, et al. Signaling from protease-activated receptor-1 inhibits migration and invasion of breast cancer cells. Can Res. 2001;61(15):5933.

26. Ashida $\mathrm{K}$, et al. Expression of e-cadherin, a-catenin, $\beta$-catenin, and CD44 (standard and variant isoforms) in human cholangiocarcinoma: an immunohistochemical study. Hepatology. 2010;27(4):974.

27. Bukholm IK, et al. E-cadherin and $\alpha-, \beta-$, and $\gamma$-catenin protein expression in relation to metastasis in human breast carcinoma. J Pathol. 2015;185(3):262-6. 
28. Jr LW, Fu R, Banko M. Growth factor-induced transcription via the serum response element is inhibited by cyclic adenosine 3',5'-monophosphate in MCF-7 breast cancer cells. Endocrinology. 1997;138(6):2219.

29. Siegel R, et al. Cancer statistics, 2014. CA Cancer J Clin. 2014;64(1):9-29.

30. Tang J, et al. A novel biomarker Linc00974 interacting with KRT19 promotes proliferation and metastasis in hepatocellular carcinoma. Cell Death Dis. 2014;5(12):e1549.

31. Rutnam ZJ, et al. The pseudogene TUSC2P promotes TUSC2 function by binding multiple microRNAs. Nat Commun. 2011;5(1):2914.

32. Chen J, et al. Competing endogenous RNA network analysis identifies critical genes among the different breast cancer subtypes. Oncotarget. 2017;8(6):10171-84.

33. Zhou X, Liu J, Wang W. Construction and investigation of breast-cancerspecific ceRNA network based on the mRNA and miRNA expression data. IET Syst Biol. 2014;8(3):96-103.

34. Zhou S, et al. Systematical analysis of IncRNA-mRNA competing endogenous RNA network in breast cancer subtypes. Breast Cancer Res Treat. 2018;169(2):267-75.

35. LiT, et al. Upregulation of long noncoding RNA ZEB1-AS1 promotes tumor metastasis and predicts poor prognosis in hepatocellular carcinoma. Oncogene. 2016;35(12):1575.

36. Yuan SX, et al. Antisense long non-coding RNA PCNA-AS1 promotes tumor growth by regulating proliferating cell nuclear antigen in hepatocellular carcinoma. Cancer Lett. 2014;349(1):87-94.

37. Li Z, et al. Comprehensive analysis of differential co-expression patterns reveal transcriptional dysregulation mechanism and identify novel prognostic IncRNAs in esophageal squamous cell carcinoma. Oncotargets Ther. 2017;10:3095-105.

38. Xing Y, et al. Comprehensive analysis of differential expression profiles of mRNAs and IncRNAs and identification of a 14-IncRNA prognostic signature for patients with colon adenocarcinoma. Oncol Rep. 2018;39(5):2365-75.

39. Wang $H$, et al. LncRNAs expression profiling in normal ovary, benign ovarian cyst and malignant epithelial ovarian cancer. Sci Rep. 2016;6:38983.

40. Guttilla IK, White BA. Coordinate regulation of FOXO1 by miR-27a, miR-96, and miR-182 in breast cancer cells. J Biol Chem. 2009;284(35):23204-16.

41. Li P, et al. MiR-183/-96/-182 cluster is up-regulated in most breast cancers and increases cell proliferation and migration. Breast Cancer Res. 2014;16(6):473.

42. Yan $L X$, et al. MicroRNA miR-21 overexpression in human breast cancer is associated with advanced clinical stage, lymph node metastasis and patient poor prognosis. RNA. 2008;14(11):2348.

43. Zhou X, et al. Decreased miR-204 in H. pylori-associated gastric cancer promotes cancer cell proliferation and invasion by targeting SOX4. PLoS ONE. 2014;9(7):e101457.

44. Butrym A, et al. Low expression of microRNA-204 (miR-204) is associated with poor clinical outcome of acute myeloid leukemia (AML) patients. J Exp Clin Cancer Res. 2015;34(1):1-5.

45. Iwaya T, et al. Downregulation of miR-144 is associated with colorectal cancer progression via activation of mTOR signaling pathway. Carcinogenesis. 2012;33(12):2391-7.
Ready to submit your research? Choose BMC and benefit from:

- fast, convenient online submission

- thorough peer review by experienced researchers in your field

- rapid publication on acceptance

- support for research data, including large and complex data types

- gold Open Access which fosters wider collaboration and increased citations

- maximum visibility for your research: over $100 \mathrm{M}$ website views per year

At BMC, research is always in progress.

Learn more biomedcentral.com/submissions 